\title{
Modelagem dos efeitos do vento na uniformidade da irrigação por aspersão: Aspersores de tamanho médio ${ }^{1}$
}

\author{
Lessandro C. Faria ${ }^{2}$, Samuel Beskow ${ }^{3}$, Alberto Colombo $^{4} \&$ Henrique F. E. de O liveira ${ }^{4}$ \\ RESU M O \\ 0 vento afeta a distribuição de água aplicada por aspersores, podendo tornar um determinado sistema \\ de irrigação ineficiente. N estas condições, a modelagem pode facilitar as avaliações de sistemas \\ convencionais de aspersão, antes mesmo de sua implantação no campo. Neste estudo foi avaliada a \\ aplicabilidade do modelo semiempírico de Richards \& W eatherhead para aspersores de tamanho médio, \\ modelos Agropolo/NY e Naan/5024, em diferentes condições de vento. Os valores do Coeficiente de \\ Uniformidade de Christiansen (CUC), obtidos com dados de ensaios de campo e em simulações \\ computacionais, foram comparados por meio de diversos índices resultando em valores de $R^{2}=0,88$, \\ desvio absoluto médio (DAM) $=4,75 \%$ e índice de desempenho $(\mathrm{c})=0,91$, para aspersores operando \\ isoladamente, para linhas laterais de aspersores, valores de $R^{2}=0,78, D A M=4,1 \%$ e $c=0,76$. \\ Demonstrou-se, assim, a aplicabilidade do modelo de Richards $\&$ W eatherhead frente à predição da \\ uniformidade de aplicação de água de sistemas convencionais de irrigação operando com aspersores \\ de tamanho médio em condições de vento, o que reduz o esforço e o tempo requerido em trabalhos de \\ campo.
}

Palavras-chave: aspersão convencional, modelo semiempírico, ensaios de campo

\section{Modeling of wind effects on water application uniformity of sprinkler irrigation: Medium-sized sprinklers}

\begin{abstract}
A B ST RACT
The wind has influence on sprinkler water distribution and it can make a given irrigation system inefficient. Under this circumstance, the use of mathematical models can ease evaluations of conventional sprinkler irrigation systems, even before they are set up in the field. The aim of this study was to evaluate the applicability of the Richards \& W eatherhead semi-empirical model using medium-sized sprinklers (Agropolo/NY and Naan/5024) under windy conditions. The Christiansen coefficient of uniformity (CU C) values, based on in-field sprinkler tests, were compared to simulated values, resulting in the following indices: (i) for individual solid set sprinkler system $R^{2}=0.88$, average absolute deviation $(A A D)=$ $4.75 \%$, and performance index (c) $=0.91$; (ii) for block sprinkler system $R^{2}=078, A A D=4.1 \%$, and $c=0.76$. These results emphasize the applicability of the Richards $\&$ W eatherhead model on water application uniformity prediction for conventional sprinkler systems operating with medium-sized sprinklers, reducing the efforts and the time required in field trials.
\end{abstract}

Key words: conventional sprinkler irrigation, semi-empirical model, in-field tests

${ }^{1}$ Parte da Tese de Doutorado do primeiro autor, apresentada à Universidade Federal de Lavras, com bolsa da CAPES DER/FAEM/UFPel,CEP 96160-000, Pelotas, RS. Fone: (53) 3275-7259. E-mail: lessandro.faria@ufpel.edu.br

3 CDTec/Eng.Hídrica/U FPel, CEP 96010-610, Pelotas, RS. Fone: (53) 3921-1240. E-mail: samuel.beskow @ufpel.edu.br

DEG/U FLA, CP 3037, CEP 37200-000, Lavras, MG. Fone: (35) 3829 1388. E-mail: acolombo@ufla.com; henrique.ellias@hotmail.com 


\section{INTRODUÇÃO}

Os sistemas de irrigação por aspersão tipo portátil são de plena utilização no Brasil, exigindo grande número de aspersores de pequeno e médio porte, irrigando diferentes culturas. A uniformidade de aplicação de água desses sistemas depende de diversos fatores, como: regulagens mecânicas do aspersor, forma geométrica do perfil de distribuição de água, disposição e espaçamento entre aspersores, pressão de serviço e velocidade e direção do vento (Coelho \& Folegatti, 1998).

Conforme Holzapfel et al. (2007) e Justi et al. (2010), em sistemas convencionais de aspersão o jato de água sofre influência direta do vento e, segundo Oliveira et al. (2009), é complexo o estudo dos efeitos do vento em condições de campo, razão pela qual é bastante utilizada a modelagem computacional da distribuição de água.

Atualmente, para aspersores de tamanho pequeno e médio, tem-se utilizado modelos baseados em teorias balísticas, visando simular a distribuição espacial de água em condições de vento. O modelo SIRIAS (Carrión et al., 2001), que necessita de ensaios de aspersores operando com e sem vento na calibração de seus parâmetros, tem sido utilizado com êxito na simulação de aspersores de tamanho médio (Montero et al., 2001; Playán et al., 2006) e de microaspersores (Conceição \& Coelho, 2003).

Além de modelos físicos baseados em teorias balísticas para simulação da distribuição de água de aspersores, diversos autores fazem sua opção por modelos empíricos de simulação, devido principalmente ao número reduzido de parâmetros de ajuste.

Em condições de vento o modelo de Richards \& Weatherhead (1993) é, hoje, bastante empregado em simulações com canhões hidráulicos, operando em sistemas autopropelidos (Granier et al., 2003; Newell, 2003; Smith et al., 2008; Prado \& Colombo, 2010a; 2010b) bem como em sistemas convencionais de aspersão (Faria et al., 2009). Esses estudos, no entanto, são voltados para aspersores que operam com altas pressões (canhões hidráulicos), sem que se leve em consideração a aplicabilidade deste modelo para prever a uniformidade de distribuição de água de aspersores de menor porte, como sugerido por Granier et al. (2003).

Para preencher esta lacuna e tendo em vista as vantagens decorrentes da utilização da modelagem computacional na simulação dos efeitos do vento sobre a aplicação de água de aspersores, o trabalho teve como objetivos: (i) ajustar, para aspersores de tamanho médio, os seis parâmetros empíricos utilizados no modelo de Richards \& Weatherhead (1993) e (ii) avaliar a aplicabilidade deste modelo na previsão da uniformidade de distribuição de água dos aspersores Agropolo/ NY e Naan/5024, operando em condições de vento.

\section{Material E MÉTODOS}

\section{Ensaios de distribuição de água}

Foram utilizados dados de distribuição espacial de água de 57 ensaios realizados com aspersores modelos Agropolo/NY e Naan/5024, cujo ângulo de inclinação do jato de água é de $12^{0}$, operando isoladamente em diferentes condições de vento (Tabela 1). Os ensaios foram conduzidos em área gramada próximo ao Laboratório de Hidráulica da Universidade Federal de Lavras (UFLA), Lavras, MG (Beskow et al., 2011), de acordo o método da malha, descrito na norma ISO 7749-2 (ISO, 1990).

Tabela 1. Velocidade média, direção do vento e combinações de bocal e pressões de serviço dos ensaios de campo com aspersores operando isoladamente

\begin{tabular}{|c|c|c|}
\hline \multicolumn{3}{|c|}{ Pressão de serviço (kPa) } \\
\hline 194 & 245 & 294 \\
\hline \multicolumn{3}{|c|}{ Velocidade ( $\mathrm{m} \mathrm{s}^{-1}$ ) / Direção do vento (graus) } \\
\hline \multicolumn{3}{|c|}{ Agropolo/NY (Bocal = 3,5 mm) } \\
\hline $0,23 / 239,10$ & $0,00 / 0,00$ & $0,68 / 298,64$ \\
\hline $0,58 / 104,31$ & $0,42 / 270,99$ & $0,68 / 27,35$ \\
\hline $0,85 / 301,37$ & $0,47 / 78,88$ & $0,78 / 230,86$ \\
\hline $0,94 / 310,31$ & $0,62 / 274,43$ & $0,85 / 270,55$ \\
\hline $1,02 / 258,67$ & $0,62 / 223,85$ & $1,36 / 315,22$ \\
\hline $1,09 / 247,66$ & $0,62 / 327,90$ & $1,40 / 209,37$ \\
\hline $1,37 / 20,87$ & $0,82 / 341,13$ & $1,64 / 348,06$ \\
\hline $2,20 / 265,36$ & $1,12 / 348,34$ & $2,01 / 320,81$ \\
\hline $2,45 / 217,86$ & $1,17 / 272,94$ & $2,13 / 206,45$ \\
\hline $2,92 / 343,57$ & $1,33 / 210,79$ & - \\
\hline- & $1,46 / 273,46$ & - \\
\hline- & $1,48 / 309,89$ & - \\
\hline- & $2,08 / 279,95$ & - \\
\hline- & $2,40 / 239,64$ & - \\
\hline \multicolumn{3}{|c|}{ Naan $/ 5024$ (Bocal $=3,0 \mathrm{~mm}$ ) } \\
\hline $0,00 / 0,00$ & $0,00 / 0,00$ & $0,00 / 0,00$ \\
\hline $0,00 / 0,00$ & $0,45 / 281,22$ & $0,29 / 315,43$ \\
\hline $0,23 / 251,82$ & $0,87 / 263,66$ & $0,61 / 287,89$ \\
\hline $0,58 / 78,69$ & $1,06 / 277,48$ & $0,92 / 292,97$ \\
\hline $0,78 / 78,90$ & $1,13 / 40,03$ & $1,12 / 246,87$ \\
\hline $1,40 / 279,68$ & $1,36 / 267,33$ & $1,29 / 261,82$ \\
\hline $1,46 / 297,94$ & 1,68 / 239,31 & $2,40 / 239,64$ \\
\hline $1,46 / 255,84$ & $2,15 / 247,56$ & \\
\hline $2,05 / 254,16$ & - & - \\
\hline
\end{tabular}

Nos ensaios de distribuição de água, com duração de uma hora, utilizou-se uma malha de coletores do tipo lata de óleo (de cozinha), pintados na cor branca, com diâmetro interno de $83,5 \mathrm{~mm}$ e altura de $190 \mathrm{~mm}$, situados a $500 \mathrm{~mm}$ da superfície do solo, regularmente espaçados $2 \mathrm{~m}$; em cada ensaio a velocidade e a direção do vento, foram registradas através de uma estação agrometeorológica modelo Vantage Pro2, em intervalos regulares de $5 \mathrm{~min}$, localizada a $2 \mathrm{~m}$ de altura da superfície do solo e a $50 \mathrm{~m}$ da área irrigada.

Os volumes de água coletados ao final de cada ensaio foram convertidos em valores de intensidade de precipitação $\left(\mathrm{mm} \mathrm{h}^{-1}\right)$ e registrados em uma matriz "A", de 12 x 12. Na Figura 1 podese observar o sistema de eixos $(\mathrm{X}, \mathrm{Y})$ adotado nos ensaios de campo para identificação das coordenadas dos 144 coletores representados na matriz "A", bem como a notação do registro do ângulo $\Phi$, que descreve a direção predominante do vento em relação às linhas de coletores de água utilizados nos ensaios de campo.

Além dos 57 ensaios de distribuição de água com o aspersor operando isoladamente, foram utilizados também 24 ensaios com linhas laterais de aspersores dos modelos Agropolo/NY e Naan/5024 (Beskow et al., 2011), conforme se observa na Tabela 2. 


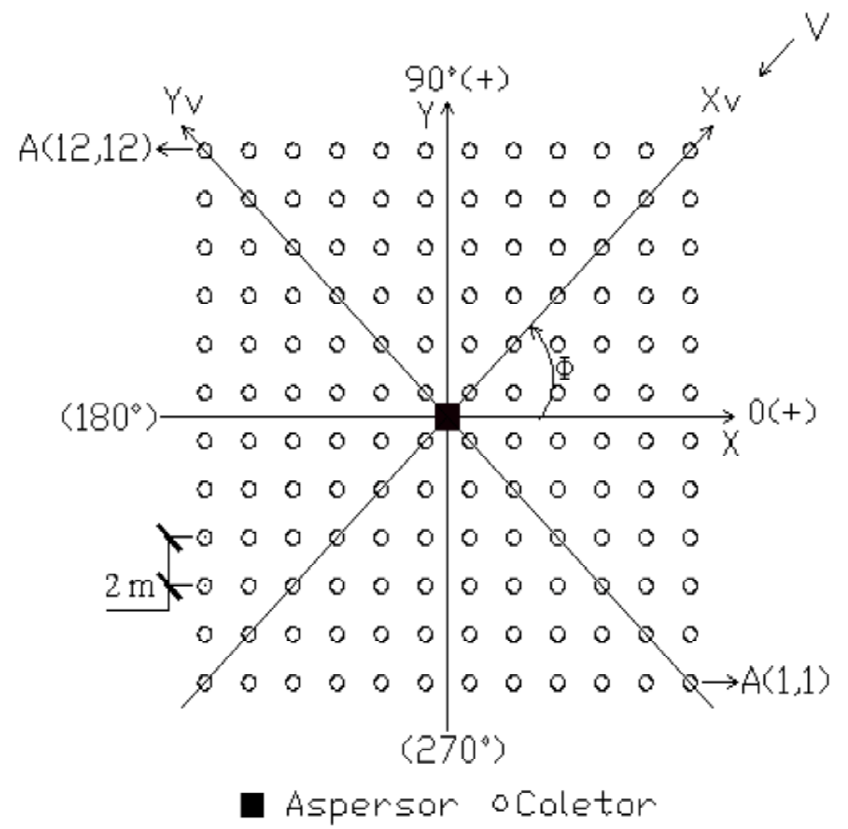

Figura 1. Esquema da malha de coletores ao redor do aspersor e inversão do eixo orientado no teste de campo $(X, Y)$ para o eixo orientado de acordo a direção predominante do vento $(\mathrm{Xv}, \mathrm{Yv})$

Tabela 2. Velocidade média e direção do vento, combinações de bocal e pressão de serviço (PS) observado em ensaios de campo com linhas laterais de aspersores

\begin{tabular}{ccc}
\hline PS & \multicolumn{2}{c}{ Vento } \\
\cline { 2 - 3 }$(\mathbf{k P a})$ & $\begin{array}{c}\text { Veloc. } \\
\left(\mathbf{m ~ s}^{-1}\right)\end{array}$ & $\begin{array}{l}\text { Direção } \\
\text { (graus) }\end{array}$ \\
194 & $\begin{array}{c}\text { Agropolo (Bocal = 3,5 mm) } \\
1,25\end{array}$ \\
194 & 1,27 & 164,89 \\
194 & 1,33 & 144,09 \\
194 & 2,30 & 152,73 \\
194 & 2,50 & 180,73 \\
245 & 1,49 & 159,18 \\
294 & 0,67 & 155,64 \\
294 & 1,13 & 220,00 \\
294 & 1,36 & 188,20 \\
294 & 3,07 & 232,30 \\
& 167,73 \\
245 & Naan (Bocal $=3,0 \mathrm{~mm})$ & \\
245 & 1,04 & 239,91 \\
245 & 1,53 & 253,55 \\
245 & 2,02 & 276,82 \\
245 & 2,11 & 167,00 \\
294 & 3,08 & 180,09 \\
294 & 0,55 & 190,29 \\
294 & 0,87 & 137,30 \\
294 & 1,78 & 276,50 \\
343 & 2,15 & 164,73 \\
343 & 1,23 & 163,82 \\
343 & 1,29 & 293,73 \\
343 & 1,69 & 183,55 \\
343 & 1,91 & 294,64 \\
& 2,26 & 181,18 \\
\hline
\end{tabular}

Nesses ensaios os aspersores foram dispostos em duas linhas laterais no espaçamento de 12 x 12 m contendo dois aspersores em cada linha lateral. Os 36 coletores foram dispostos de maneira a formar quadrados de $2 \mathrm{~m}$ de lado. Os referidos ensaios foram de $50 \mathrm{~min}$, com duração média de 10 min para a realização das leituras dos volumes de água nos coletores. A fim de controlar a pressão de serviço no sistema durante a realização dos testes de irrigação, duas tomadas de pressão foram utilizadas, sendo uma localizada entre a válvula de gaveta e o primeiro aspersor e a outra no tubo de subida do último aspersor; por fim, a obtenção dos dados meteorológicos foi realizada da mesma maneira que nos ensaios com o aspersor operando isoladamente.

\section{Ajuste dos parâmetros empíricos do modelo de Richards \& Weatherhead (1993)}

Um programa computacional foi desenvolvido na linguagem de programação Delphi 7 (ambiente Windows), baseada no modelo de Richards \& Weatherhead (1993), para simular a distribuição espacial de água de um aspersor operando em condições de vento. A rotina desenvolvida gerou os valores de precipitação $\left(\mathrm{mm} \mathrm{h}^{-1}\right)$ ao longo de uma malha de pontos (coletores fictícios) uniformemente distribuídos ao redor do aspersor, observando-se o mesmo arranjo de coletores utilizados nos ensaios de campo (Figura 1).

Implementou-se, para o ajuste dos parâmetros empíricos (A, B, C, D, E e F) do modelo, uma rotina baseada no método Shuffled Complex Evolution (SCE-UA), proposto por Duan et al. (1992), cujo objetivo foi minimizar simultaneamente e de acordo com o recomendado por Richards \& Weatherhead (1993), a soma do quadrado dos desvios entre valores de intensidade de precipitação estimados em três malhas de ensaio geradas pelo programa e valores de intensidade de precipitação observados em três malhas de ensaios de campo.

As informações seguintes são requeridas como dados de entrada do programa computacional:

i) Dados do aspersor (Figura 2A):
a) Marca/Modelo
b) Diâmetro do bocal
c) Ângulo de saída do jato de água
d) Pressão de serviço
e) Raio de alcance na ausência de vento

ii) Dados de vento:

a) Velocidade média do vento

b) Direção média do vento em relação às coordenadas $\mathrm{x}$ e y na posição dos coletores ao redor do aspersor no sistema de eixos orientados utilizados nos ensaios de campo (Figura $1)$.

iii) Dados requeridos pelo método SCE-UA (Figura 2B):

Sendo "n" o número de parâmetros a serem ajustados $(n=6)$, cujos limites inferior e superior do espaço amostral de cada parâmetro foram - 1 e 1 . Os valores de " $\mathrm{m}, \mathrm{q}, \mathrm{p}_{\min }$, $\alpha$ e $\beta$ " utilizados, foram os recomendados por Duan et al. (1992), obtidos por:

a) p, o número de complexos

b) $\mathrm{m}$, o número de pontos em um complexo $(\mathrm{m}=2 \mathrm{n}+1$, então $m=13$ )

c) q, o número de pontos em um subcomplexo $(q=n+1$, então $q=7$ ) 
A.

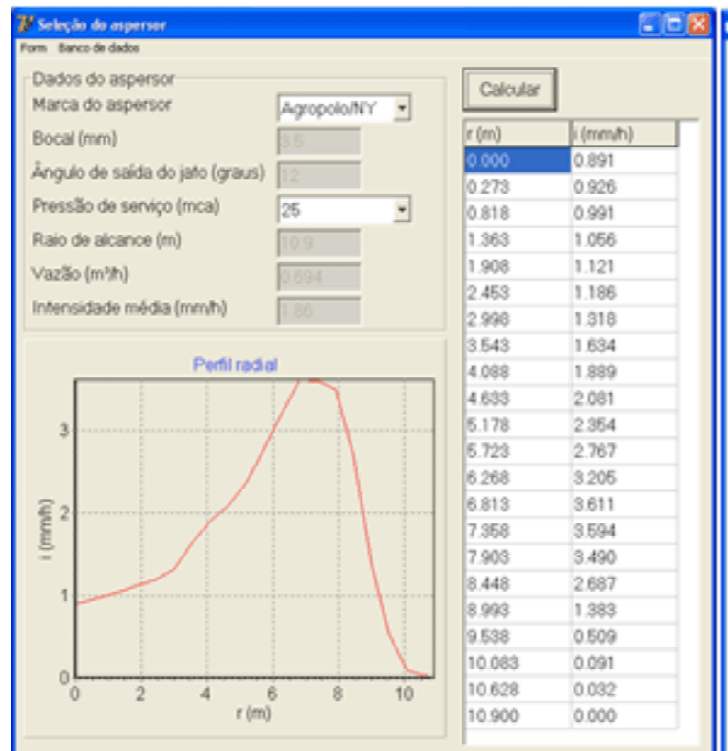

B.

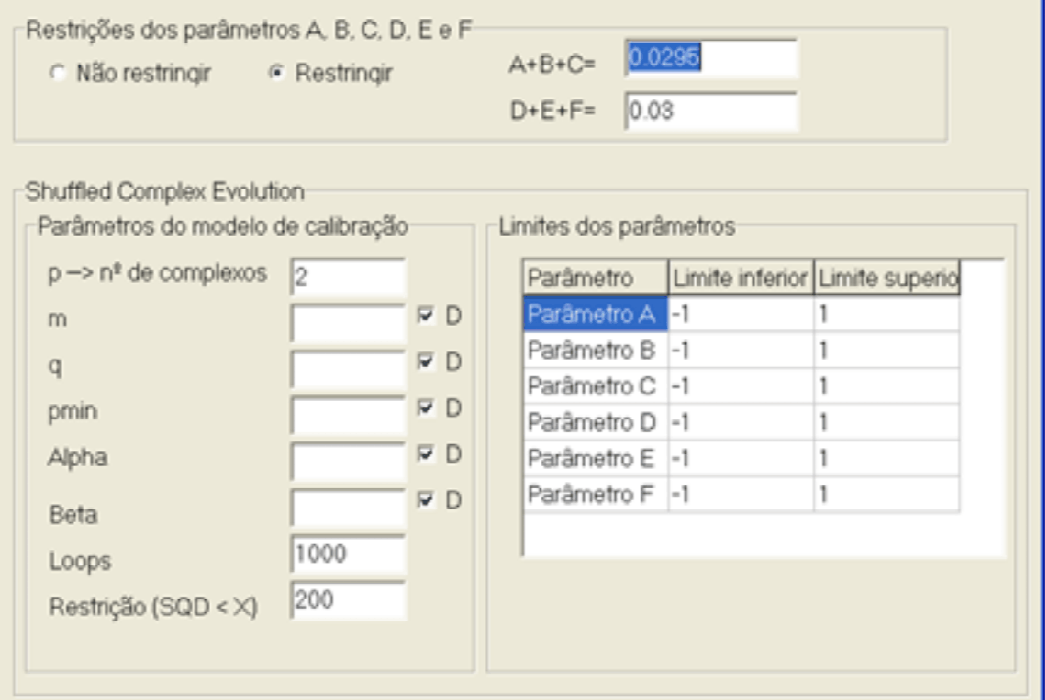

Figura 2. Informações requeridas como dados de entrada do programa computacional: (A) D ados do aspersor e (B) Dados requeridos pelo método SCE-U A

d) $\mathrm{p}_{\min }$, o número mínimo de complexos $\left(1 \leq \mathrm{p}_{\min } \leq \mathrm{p}\right)$

e) $\alpha$, o número consecutivo de valores gerados por cada subcomplexo $(\mathrm{a}=1)$

f) $\beta$, o número de evoluções de cada complexo $(\beta=\mathrm{m}=13)$

De acordo com a Figura 2B, tem-se a opção de restringir os valores das somas das constantes empíricas do modelo, os quais definem os efeitos da deriva do vento $(A+B+C)$ e a redução do alcance do jato $(\mathrm{D}+\mathrm{E}+\mathrm{F})$, valores obtidos pela análise das dimensões do alcance do jato do aspersor (Faria, 2011). Assim, quando se restringem esses valores, tem-se maior facilidade de convergência para o valor mínimo da função objetivo dos valores das seis constantes empíricas do modelo. Além disso, é necessária, para as simulações, a curva de aplicação de água do aspersor operando na ausência de vento, contendo vinte pares de valores de intensidade de precipitação e distância ao aspersor (adimensional) permanecendo esses valores disponibilizados no banco de dados do programa (conforme observado na Figura 2A).

Os dados de entrada foram processados de acordo com os seguintes passos:

i) cálculo, com base no valor do raio de alcance sem vento ( $\mathrm{R}$ em $\mathrm{m})$, do valor não ponderado $(\mathrm{A}=(\mathrm{A} / \mathrm{R}) \cdot \mathrm{R} . . ., \mathrm{F}=(\mathrm{F} / \mathrm{R}) \cdot \mathrm{R})$ das constantes empíricas do modelo de Richards \&Weatherhead (1993).

ii) conversão das coordenadas $(\mathrm{x}, \mathrm{y})$ da posição do coletor tomadas no sistema de eixos dos ensaios de campo, para as coordenadas $\left(\mathrm{x}_{\mathrm{v}}, \mathrm{y}_{\mathrm{v}}\right)$ do sistema de eixos orientado contra $\mathrm{o}$ sentido do vento definido no modelo de Richards \& Weatherhead (1993). Esta conversão é realizada por meio das Eq. 1 e 2, que se baseiam no ângulo do vento $\Phi$, esquematizado na Figura 1.

$$
\mathrm{x}_{\mathrm{v}}=\mathrm{x} \cdot \cos (\Phi)+\mathrm{y} \cdot \operatorname{sen}(\Phi)
$$

$$
\mathrm{y}_{\mathrm{v}}=\mathrm{y} \cdot \cos (\Phi)-\mathrm{x} \cdot \operatorname{sen}(\Phi)
$$

iii) cálculo, por meio de um método iterativo, das coordenadas $\left(\mathrm{x}_{\mathrm{v} 0}, \mathrm{y}_{\mathrm{v} 0}\right)$ do ponto de impacto sem vento, que resultam em um ponto de impacto com vento coincidente com a posição do coletor em questão, que tem coordenadas $\left(\mathrm{x}_{\mathrm{v}}, \mathrm{y}_{\mathrm{v}}\right)$. $\mathrm{O}$ processo iterativo para determinação das coordenadas $\left(\mathrm{x}_{\mathrm{v} 0}, \mathrm{y}_{\mathrm{v} 0}\right)$ tem início com $\mathrm{x}_{\mathrm{v} 0}=\mathrm{x}_{\mathrm{v}}, \mathrm{e}_{\mathrm{v} 0}=\mathrm{y}_{\mathrm{v}}$, terminando quando o valor absoluto das correções das estimativas de $\mathrm{x}_{\mathrm{v} 0} \mathrm{e}_{\mathrm{v} 0}$ é menor que $0,001 \mathrm{~m}$. Para acelerar a convergência do processo iterativo, o método de Newton, descrito por Burden \& Faires (2003), foi incorporado ao programa computacional e aplicado ao seguinte sistema de equações simultâneas dadas no modelo Richards \& Weatherhead (1993), para descrever as coordenadas do ponto de impacto na ausência de vento $\left(\mathrm{x}_{\mathrm{v} 0}, \mathrm{y}_{\mathrm{v} 0}\right)$ e as coordenadas do ponto de impacto na presença de vento $\left(\mathrm{x}_{\mathrm{v}}, \mathrm{y}_{\mathrm{v}}\right)$.

$$
\begin{aligned}
& x_{v}=x_{v 0}-\left[A+B \cdot(r / R)+C \cdot(r / R)^{2}\right] \cdot V-\left[D \cdot(r / R)+E \cdot(r / R)^{2}+\ldots\right. \\
& \left.F \cdot(r / R)^{3}\right] \cdot V \cdot \sqrt{\operatorname{sen}^{2} e \cdot\left(\frac{x_{v 0}}{r}\right)^{2}+\left(\frac{y_{v 0}}{r}\right)^{2}} \cdot \frac{x_{v 0}}{r} \\
& y_{v}=y_{v 0}-\left[D \cdot(r / R)+E \cdot(r / R)^{2}+F \cdot(r / R)^{3}\right] \cdot V \cdot \sqrt{\operatorname{sen}^{2} e \cdot\left(\frac{x_{v 0}}{r}\right)^{2}+\left(\frac{y_{v 0}}{r}\right)^{2}} \cdot \frac{y_{v 0}}{r}
\end{aligned}
$$

em que:

$\mathrm{x}_{\mathrm{v}}, \mathrm{y}_{\mathrm{v}}$ - coordenadas do ponto de impacto da água em condições de vento $(\mathrm{m})$

$\mathrm{x}_{\mathrm{v} 0}, \mathrm{y}_{\mathrm{v} 0}$ - coordenadas do ponto de impacto da água na ausência de vento $(\mathrm{m})$

$\mathrm{r}=\left(\mathrm{x}_{\mathrm{v} 0}{ }^{2}+\mathrm{y}_{\mathrm{v} 0}{ }^{2}\right)^{1 / 2}-$ distância radial do aspersor ao ponto de impacto sem vento $(\mathrm{m})$

$\mathrm{R}$ - raio de alcance do aspersor operando na ausência de vento $(\mathrm{m})$; 
$\mathrm{V}$ - velocidade do vento $\left(\mathrm{m} \mathrm{s}^{-1}\right)$

e - ângulo de saída do jato de água em relação ao plano horizontal (graus)

A, B, C, D, E e F - parâmetros empíricos do modelo

iv) cálculo da taxa da aplicação, $\mathrm{I}_{0}$, na ausência de vento, no ponto de coordenada $\mathrm{x}_{\mathrm{v} 0}$ e $\mathrm{y}_{\mathrm{v} 0}$, que corresponde à distância radial $\left.\mathrm{r}=\left(\mathrm{x}_{\mathrm{v} 0}{ }^{2}+\mathrm{y}_{\mathrm{v} 0}\right)^{2}\right)^{1 / 2}$, utilizando-se as informações do perfil radial de aplicação de água.

v) cálculo da taxa da aplicação $I_{v}$ em $x_{v}, y_{v}$ na presença de vento por meio da Eq. 5:

$$
\mathrm{I}_{\mathrm{v}}=\frac{\mathrm{I}(\mathrm{r} / \mathrm{R})_{0}}{\left(\frac{\partial \mathrm{x}_{\mathrm{v}}}{\partial \mathrm{x}_{\mathrm{v} 0}} \cdot \frac{\partial \mathrm{y}_{\mathrm{v}}}{\partial \mathrm{y}_{\mathrm{v} 0}}\right)}
$$

em que:

$\mathrm{I}_{\mathrm{v}} \quad$ - intensidade de aplicação de água em $\mathrm{x}_{\mathrm{v}}, \mathrm{y}_{\mathrm{v}}$ (condições de vento, $\mathrm{mm} \mathrm{h}^{-1}$ )

$\mathrm{I}(\mathrm{r} / \mathrm{R})_{0}$ - intensidade de aplicação de água em $\mathrm{x}_{\mathrm{v} 0}, \mathrm{y}_{\mathrm{v} 0}$, (baseada na curva de aplicação de água do aspersor operando na ausência de vento, $\mathrm{mm} \mathrm{h}^{-1}$ )

$\left(\partial \mathrm{x}_{\mathrm{v}} / \partial \mathrm{x}_{\mathrm{v} 0}\right)$ e $\left(\partial \mathrm{y}_{\mathrm{v}} / \partial \mathrm{y}_{\mathrm{v} 0}\right)$ - obtidos diferenciando-se as Eq. 3 e 4 em relação a $x_{v 0}$ e $y_{v 0}$, respectivamente

vi) execução dos passos (ii) até (v) para todos os coletores do sistema de eixos orientados utilizados nos testes de campo.

Avaliação da modelagem da uniformidade de aplicação de água

Obtiveram-se as uniformidades em sistemas convencionais de āspersão operando com aspersores de tamanho médio Agropolo/NY e Naan/5024, nas mesmas condições observadas nos ensaios de campo e pela sobreposição das matrizes contendo os valores de intensidade de precipitação ao redor do aspersor, as quais foram obtidas por meio do mesmo programa computacional em questão. No processo de sobreposição foram considerados apenas arranjos quadrados entre aspersores espaçados de 6,12 e $18 \mathrm{~m}$.

A adequação com que as simulações realizadas permitem prever os valores observados do Coeficiente de Uniformidade de Christiansen (CUC) foi avaliada seguindo-se as recomendações de Montero et al. (2001), determinando-se o desvio absoluto médio e o valor do coeficiente de determinação $\left(\mathrm{R}^{2}\right)$ e de Conceição \& Coelho (2003), no sentido de se determinar o índice de confiança de desempenho (c), de Camargo \& Sentelhas (1997). O valor de "c" é obtido pela multiplicação do coeficiente de correlação (r) pelo índice de Willmott (d), conforme apresentado nas equações abaixo:

$$
\mathrm{c}=\mathrm{r} \cdot \mathrm{d}
$$

com:

$$
\mathrm{d}=1-\left[\sum\left(\mathrm{P}_{\mathrm{i}}-\mathrm{O}_{\mathrm{i}}\right)^{2} / \sum\left(\left|\mathrm{P}_{\mathrm{i}}-\overline{\mathrm{O}}\right|+\left|\mathrm{O}_{\mathrm{i}}-\overline{\mathrm{O}}\right|\right)^{2}\right]
$$

em que:

$$
\begin{aligned}
& \mathrm{P}_{\mathrm{i}} \text { - valor estimado } \\
& \mathrm{O}_{\mathrm{i}} \text { - valor observado } \\
& \mathrm{O} \text { - média dos valores observados }
\end{aligned}
$$

O índice de confiança de desempenho foi avaliado segundo escala proposta por Camargo \& Sentelhas (1997): Ótimo (c > 0,85); Muito Bom $(0,76 \leq \mathrm{c} \leq 0,85)$; Bom $(0,66 \leq \mathrm{c} \leq 0,75)$; Mediano $(0,61 \leq \mathrm{c} \leq 0,65)$; Sofrível $(0,51 \leq \mathrm{c} \leq 0,60)$; Mau $(0,41$ $\leq \mathrm{c} \leq 0,50)$ e Péssimo $(\mathrm{c} \leq 0,40)$.

\section{RESUltados E DisCUSSÃO}

\section{Ajuste dos parâmetros empíricos do modelo de Richards \& Weatherhead}

Os 32 ensaios de distribuição de água com ventos superiores que $0,9 \mathrm{~ms}^{1}$ permitiram a formação de 4960 conjuntos, cada um contendo três ensaios; no entanto em função da análise das dimensões da área molhada distorcida pelo vento (Faria, 2011), estabeleceu-se que valores dos coeficientes empíricos ajustados atendessem às seguintes restrições: $A / R+B / R+C / R$ $=0,0295 \mathrm{sm}^{-1} \mathrm{e} \mathrm{D} / \mathrm{R}+\mathrm{E} / \mathrm{R}+\mathrm{F} / \mathrm{R}=0,0300 \mathrm{~s} \mathrm{~m}^{-1}$ (Figura $2 \mathrm{~B}$ ); desta forma, diversos conjuntos de três ensaios de campo foram submetidos ao processo de ajuste dos parâmetros, até que um conjunto contendo três ensaios de campo do aspersor Agropolo/NY com bocal de 3,5 mm e pressão de $245 \mathrm{kPa}$ : (i) vento de $1,46 \mathrm{~m} \mathrm{~s}^{-1}$ na direção de $273,46^{\circ}$; (ii) vento de $2,08 \mathrm{~m} \mathrm{~s}^{-1}$ na direção de $279,95^{\circ}$ e (iii) vento de $2,40 \mathrm{~m} \mathrm{~s}^{-1}$ na direção de $239,64^{\circ}$, fornecesse valores de parâmetros empíricos ajustados os quais atendiam ao critério estabelecido.

$\mathrm{O}$ valor individual dos coeficientes ajustados obtidos pelo programa (Figura 3) e a proximidade da soma destes coeficientes com a meta de desejada, foram: $\mathrm{A} / \mathrm{R}=0,1411 \mathrm{~s} \mathrm{~m}^{-1} ; \mathrm{B} / \mathrm{R}=-0,3213$ $\mathrm{s} \mathrm{m}^{-1}$ e C/R $=0,2097 \mathrm{~s} \mathrm{~m}^{-1}$ que resultam em: 0,0295/0,0295 = 1,0 e $\mathrm{D} / \mathrm{R}=0,0635 \mathrm{~s} \mathrm{~m}^{-1} ; \mathrm{E} / \mathrm{R}=-0,2002 \mathrm{~s} \mathrm{~m}^{-1} \mathrm{e} / \mathrm{R}=0,1667 \mathrm{~s} \mathrm{~m}^{-1}$ que resultam em: $0,03 / 0,03=1,0$.

A adequação dos parâmetros do ajuste pode ser avaliada na Figura 4, que compara mapas de contorno das taxas de aplicação de água $\left(\mathrm{mm} \mathrm{h}^{-1}\right)$ obtidas nos ensaios de campo com mapas gerados pelo programa computacional. As comparações entre as taxas observadas em campo e estimadas pelo modelo apresentaram, de acordo com o índice de confiança de Camargo $\&$ Sentelhas (1997), desempenho Ótimo (c > 0,85) ou Muito Bom $(0,76 \leq \mathrm{c} \leq 0,85)$, com valores de "c" iguais a $0,92,0,85 \mathrm{e}$ 0,77 para os ensaios i, ii e iii, respectivamente; ressalta-se entretanto que mesmo quando os modelos matemáticos não representam precisamente a distribuição de água de aspersores operando sob condições de vento, eles continuam sendo ferramentas úteis no auxílio a tomadas de decisões em projetos e na avaliação de sistemas de irrigação (Voires et al., 1987; Prado \& Colombo, 2010a).

Avaliação da modelagem da uniformidade de aplicação de água

$\mathrm{Na}$ Figura 5 tem-se a relação entre os 225 valores do Coeficiente de Uniformidade de Christiansen (CUC) obtidos por simulação dos aspersores de tamanho médio Agropolo/ 


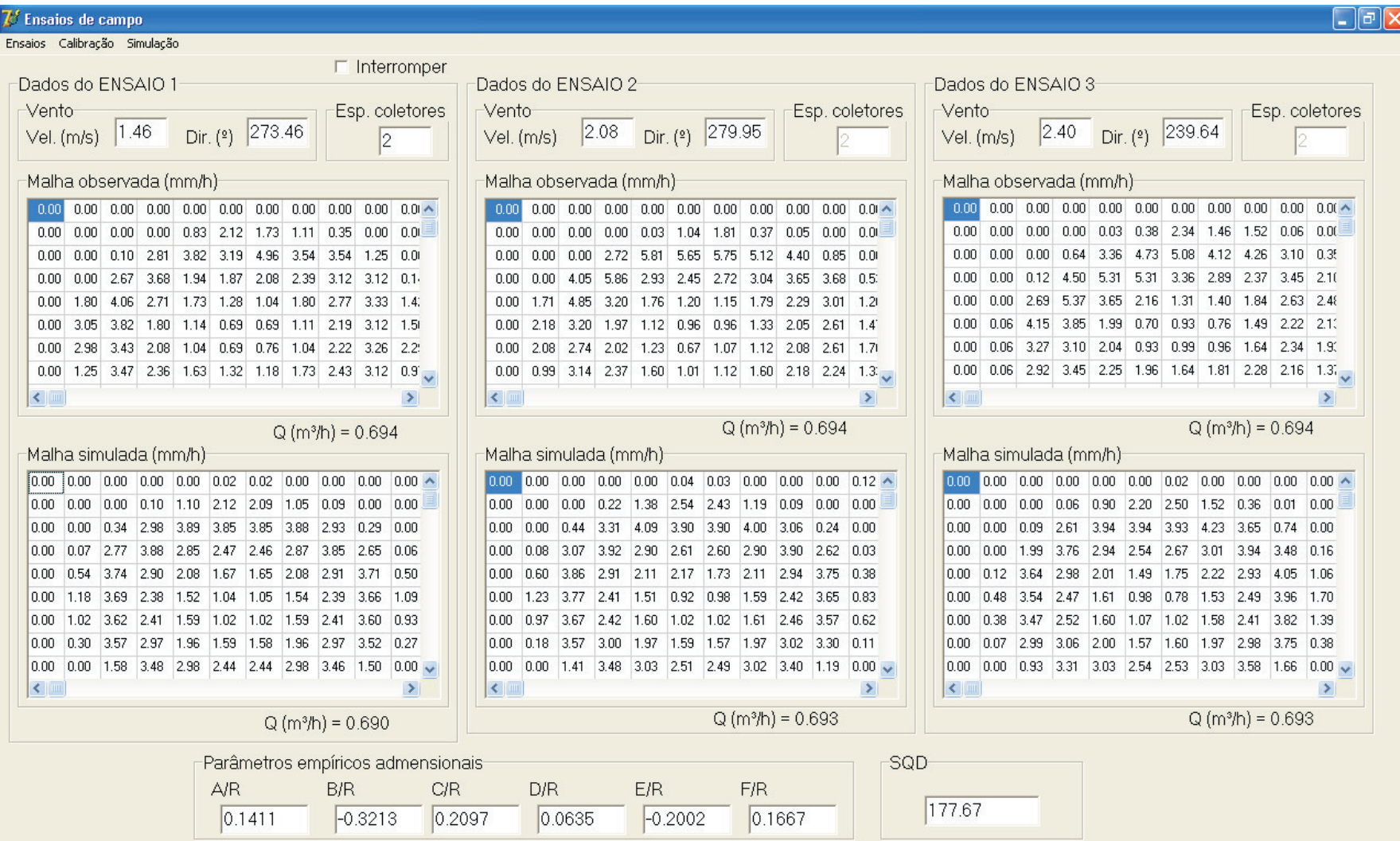

Figura 3. Dados de velocidade e direção de vento requeridos para o ajuste, valores ajustados das seis constantes empíricas (A, B, C, D, E e F) do modelo de Richards \& Weatherhead e malhas com os valores de intensidade de aplicação $\left(\mathrm{mm} \mathrm{h}^{-1}\right)$ ao redor do aspersor

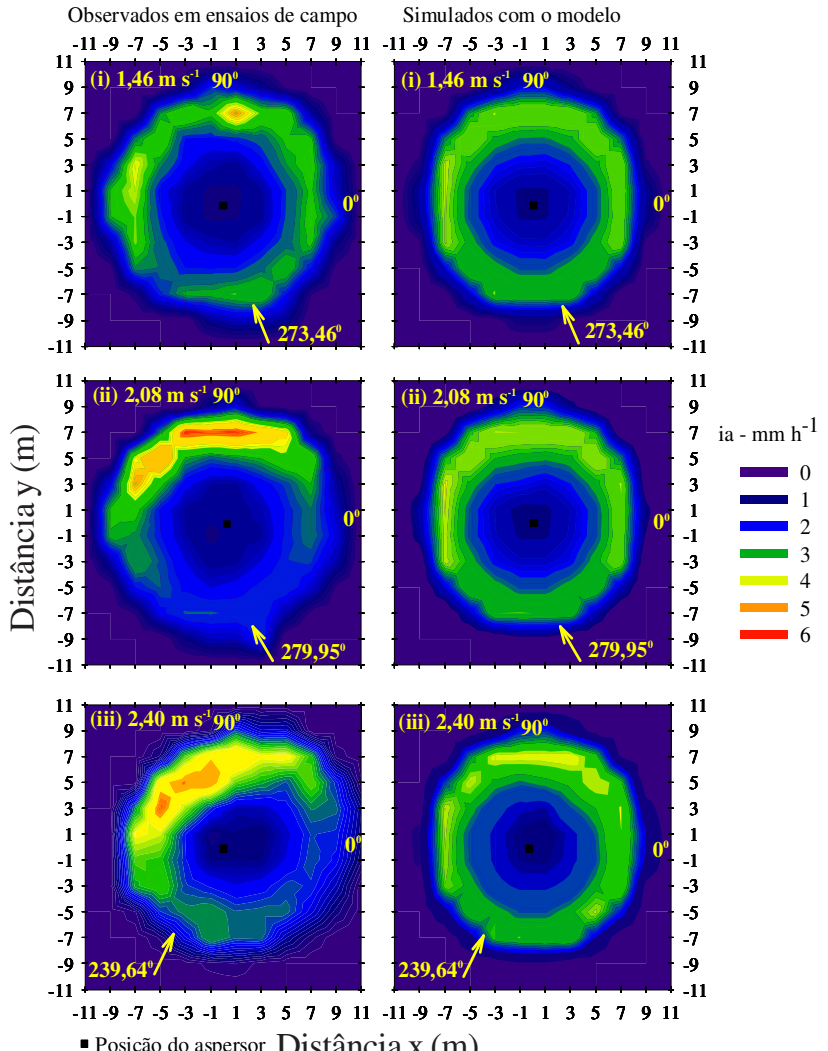

Figura 4. Superfícies de contorno das intensidades de aplicação de água do aspersor $\left(\mathrm{mm} \mathrm{h}^{-1}\right)$ nos três ensaios utilizados para o ajuste das constantes

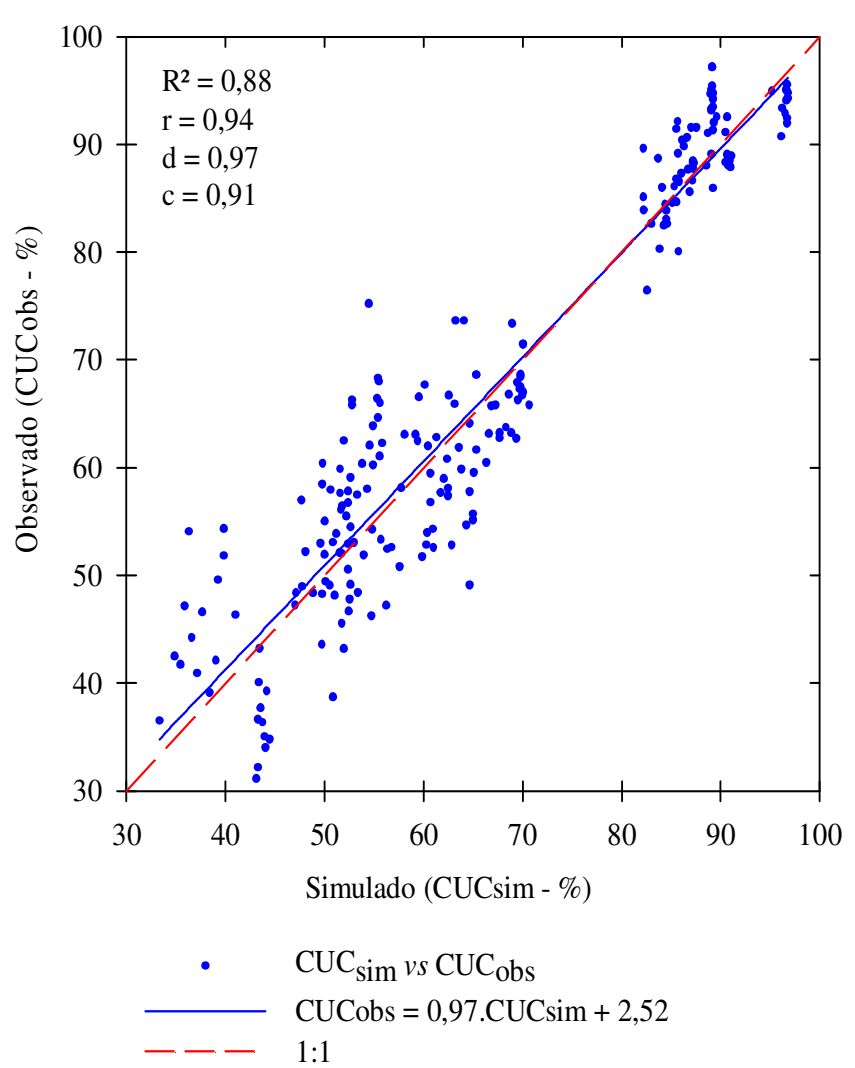

Figura 5. Relação entre os valores de Coeficiente de Uniformidade de Christiansen obtidos por simulações $\left(C \cup C_{\text {sim }}\right)$ e a partir de ensaios de campo $\left(\mathrm{CUC}_{\mathrm{obs}}\right)$ 
NY e Naan/5024 e os obtidos em ensaios de campo. Verifica-se que os pares de valores de CUC estão bem distribuídos ao redor da reta 1:1, conforme se observa pelo valor do coeficiente angular da equação de regressão $(0,97)$; a adequação desta concentração ao redor da linha 1:1 também é confirmada pelos altos valores do índice de concordância de Willmott $(\mathrm{d}=0,97)$ e do índice de confiança $(c=0,91)$, que corresponde a uma classificação do tipo: Ótimo (c>0,85).

O valor de "c" obtido na comparação de dados simulados com dados de ensaios de campo dos aspersores de tamanho médio Agropolo/NY e Naan/5024 foi superior aos obtidos por Faria et al. (2009) com o canhão hidráulico Plona/RL-250 (c = 0,78 - classificação do tipo: Muito Bom).

A diferença entre os valores do índice "c" obtidos entre canhões hidráulicos e aspersores de tamanho médio, pode ser atribuída ao número de comparações entre valores simulados e observados de CUC (319 valores com canhões hidráulicos e 225 com aspersores de média pressão). Além disso, na avaliação do canhão Plona-RL250 as velocidades de vento durante os ensaios de campo atingiram até $5 \mathrm{~m} \mathrm{~s}^{-1}$, valores superiores aos $3 \mathrm{~m} \mathrm{~s}^{-1}$ atingidos nos ensaios de campo utilizados neste estudo, considerando-se que maiores velocidades de vento proporcionam também maiores distorções do padrão de distribuição de água.

Na comparação da reta 1:1 com a linha do ajuste linear, observa-se que na região de interesse prático (CUC > 60\%) o modelo ajustado tende a produzir valores de CUC muito próximos aos observados nos ensaios de campo. O coeficiente de determinação $\left(\mathrm{R}^{2}=0,88\right)$ do ajuste linear entre valores observados e simulados de CUC resultou em um valor superior ao de Faria et al. (2009) que, com o modelo de Richards \& Weatherhead (1993), obtiveram $\mathrm{R}^{2}=0,73$ entre valores observados e simulados com o canhão Plona-RL250, e de Montero et al. (2001), que na avaliação do modelo SIRIAS, com aspersores de tamanho médio, obtiveram valores de $\mathrm{R}^{2}$ igual a 0,$51 ; 0,75 ; 0,85$ e 0,81 para os espaçamentos de $12 \times 12$; $12 \times 18 ; 18 \times 18$ e $18 \times 16$ (triangular), respectivamente.

$\mathrm{Na}$ Figura 6 são apresentados 225 valores absolutos da diferença entre valores de CUC obtidos por simulações e por ensaios de campo dos aspersores Agropolo/NY e Naan/ 5024.

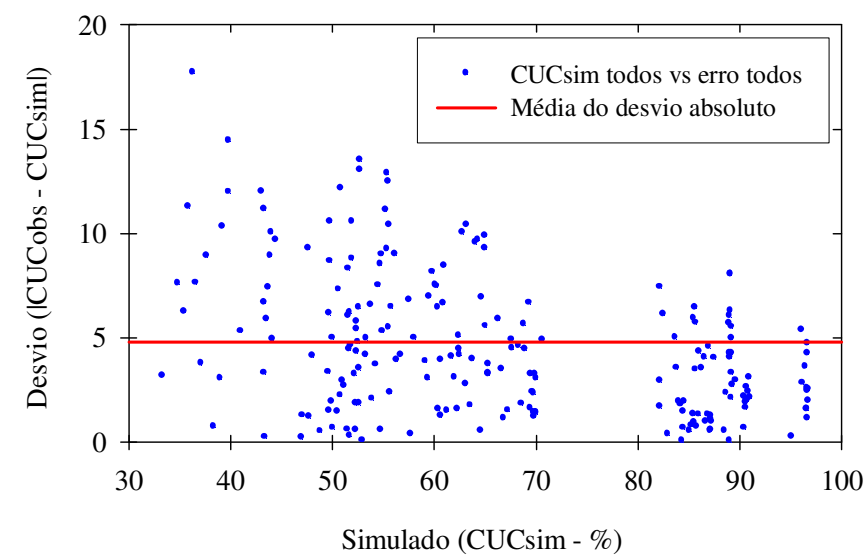

Figura 6. Diferenças absolutas entre os valores de CUC obtidos com ensaios de campo ( $\left.\mathrm{CUC}_{\mathrm{obs}}\right)$ e em simulações $\left(\mathrm{CUC}_{\mathrm{sim}}\right)$ em função dos valores de $\mathrm{CU}_{\mathrm{sim}}$
Pode-se observar, nesta figura, que o valor médio das diferenças absolutas $(4,75 \%)$, mesmo um pouco superior aos $4,1 \%$ obtidos por Faria et al. (2009) e aos 2,7\% obtidos por Montero et al. (2001), se situa abaixo do limite de 5\%, que caracteriza um desempenho classificado como "muito bom" na previsão de valores de CUC de sistemas convencionais de aspersão (Montero et al., 2001). Assim, a utilização do modelo de Richards \& Weatherhead (1993) em prever valores de uniformidade de distribuição de água de sistemas convencionais de irrigação com aspersores de tamanho médio, operando sob condições de vento, pode servir de auxílio a projetistas e irrigantes na escolha de condições operacionais e espaçamentos entre aspersores que elevem o grau de uniformidade desses sistemas, em condições reais de trabalho.

Ainda que ocorram diferenças entre valores de CUC simulados com o modelo e os observados em campo, é importante ressaltar que tais diferenças não devem ser atribuídas apenas ao modelo ajustado visto que as dificuldades de se obter, ao longo do período de duração dos ensaios de campo, o mesmo grau de estabilidade nos valores dos parâmetros climáticos considerado nas simulações.

O aumento da amplitude dos desvios correspondente aos menores valores de CUC, mostrado na Figura 6, indica que as estimativas que resultam em valores mais reduzidos de CUC são mais susceptíveis às instabilidades dos fatores climáticos. Este aspecto foi também enfatizado por Solomon (1979), ao demonstrar que, mesmo em condições de ventos fracos, os espaçamentos entre aspersores que resultam em reduzido grau de sobreposição da água aplicada por aspersores adjacentes, apresentam maior incerteza nos valores de CUC (desvio padrão de $2 \%$ para CUC perto de $90 \%$, desvio padrão de $4 \%$ para CUC próximo de $80 \%$ e desvio padrão de $6 \%$ para CUC perto de $70 \%)$.

As estimativas dos valores de uniformidade obtidas com o modelo ajustado $\left(\mathrm{CUC}_{\text {sim }}\right)$ e os valores oriundos de observações em ensaios de campo $\left(\mathrm{CUC}_{\mathrm{obs}}\right)$, para diferentes espaçamentos entre aspersores dispostos em arranjos quadrados, podem ser observados na Figura 7. As condições operacionais dos ensaios apresentados nesta figura são as mesmas utilizadas para ajustar os valores dos parâmetros empíricos do modelo de Richards \& Weatherhead (1993).

Observa-se que o comportamento da uniformidade de aplicação de água em função do aumento do espaçamento entre aspersores, foi representado adequadamente nas três condições de velocidade e direção de vento (Figura 7).

Buscou-se também, além da análise das estimativas da uniformidade de distribuição de água a partir de ensaios de campo de aspersores isolados, avaliar a capacidade do modelo de Richards \& Weatherhead (1993) em prever a uniformidade de distribuição de água de aspersores operando em linhas laterais.

$\mathrm{Na}$ Figura 8A pode-se observar a relação entre 24 valores de CUC gerados a partir de simulações baseadas no modelo de Richards \& Weatherhead (1993), ajustado para aspersores de tamanho médio (Agropolo/NY e Naan/5024) e 24 valores de CUC obtidos a partir de ensaios de campo com linhas laterais dos mesmos aspersores dispostos no espaçamento $12 \times 12 \mathrm{~m}$. 

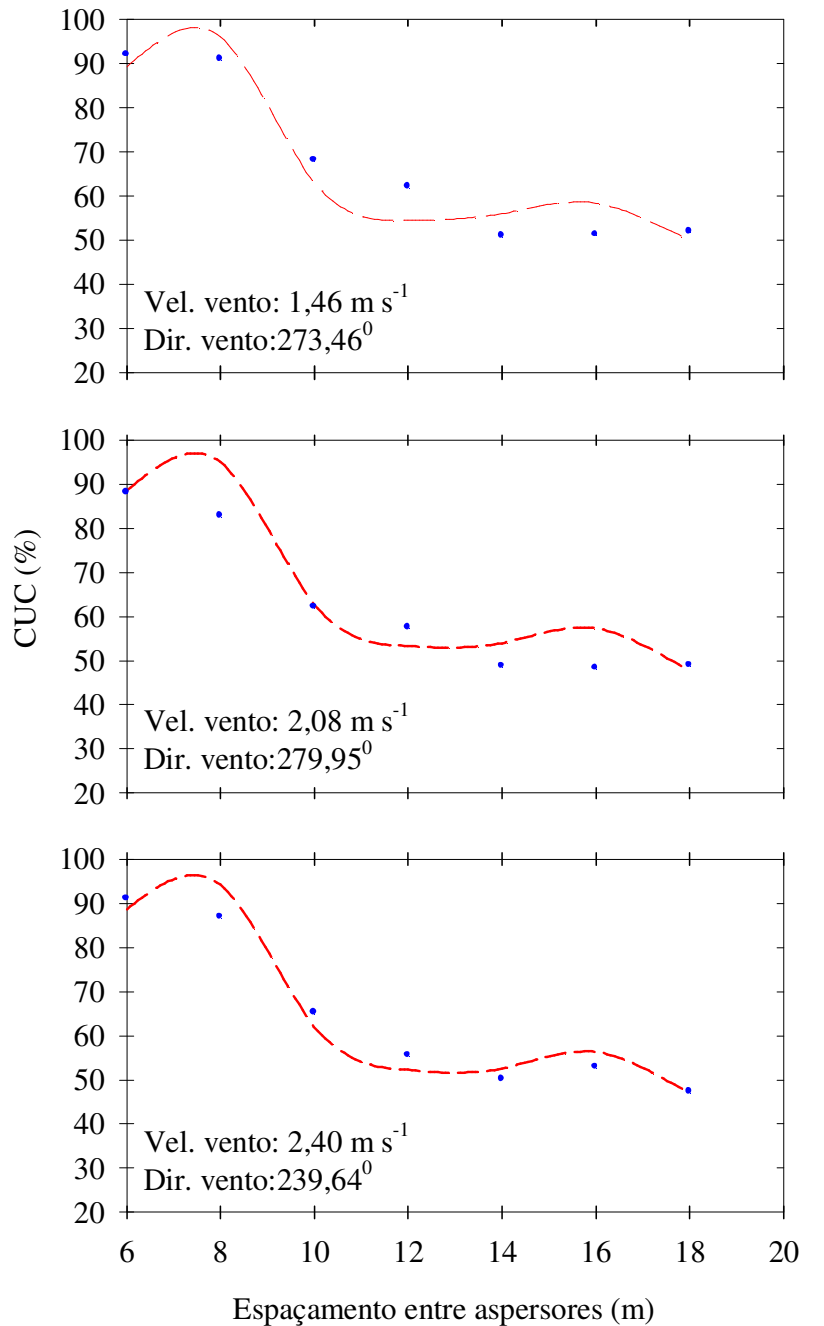

\section{- - CUC simulado \\ CUC observado}

Figura 7. Valores de CUC (\%) para diferentes espaçamentos quadrados entre aspersores Agropolo

Observa-se, na Figura 8A, que para os pares de valores de CUC (observados e simulados) inferiores a $60 \%$, ocorre uma dispersão maior dos pontos, em relação à reta 1:1, conforme previsto por Solomon (1979). No entanto, para CUC > 60\% os pares de valores se encontram mais próximos da reta 1:1. O valor do índice de confiança (c) foi igual a 0,76, classificado como sendo Muito Bom. Além disso, o valor do ajuste linear $\left(\mathrm{R}^{2}=0,78\right)$ permanece dentro dos limites observados na literatura (Montero et al., 2001; Faria et al., 2009).

Constata-se, pela Figura 8A, que o modelo ajustado tende a subestimar os valores de CUC, para aspersores operando em linhas laterais. Este comportamento pode ser atribuído ao fato de que se cria, quando vários aspersores operam simultaneamente, um microclima entre eles, que diminui as perdas de água por evaporação e arraste, quando comparados com ensaios de aspersores operando isoladamente (Ortega et al., 2000), cuja conseqüência são os maiores valores observados de uniformidade em relação aos simulados (Beskow et al., 2011).
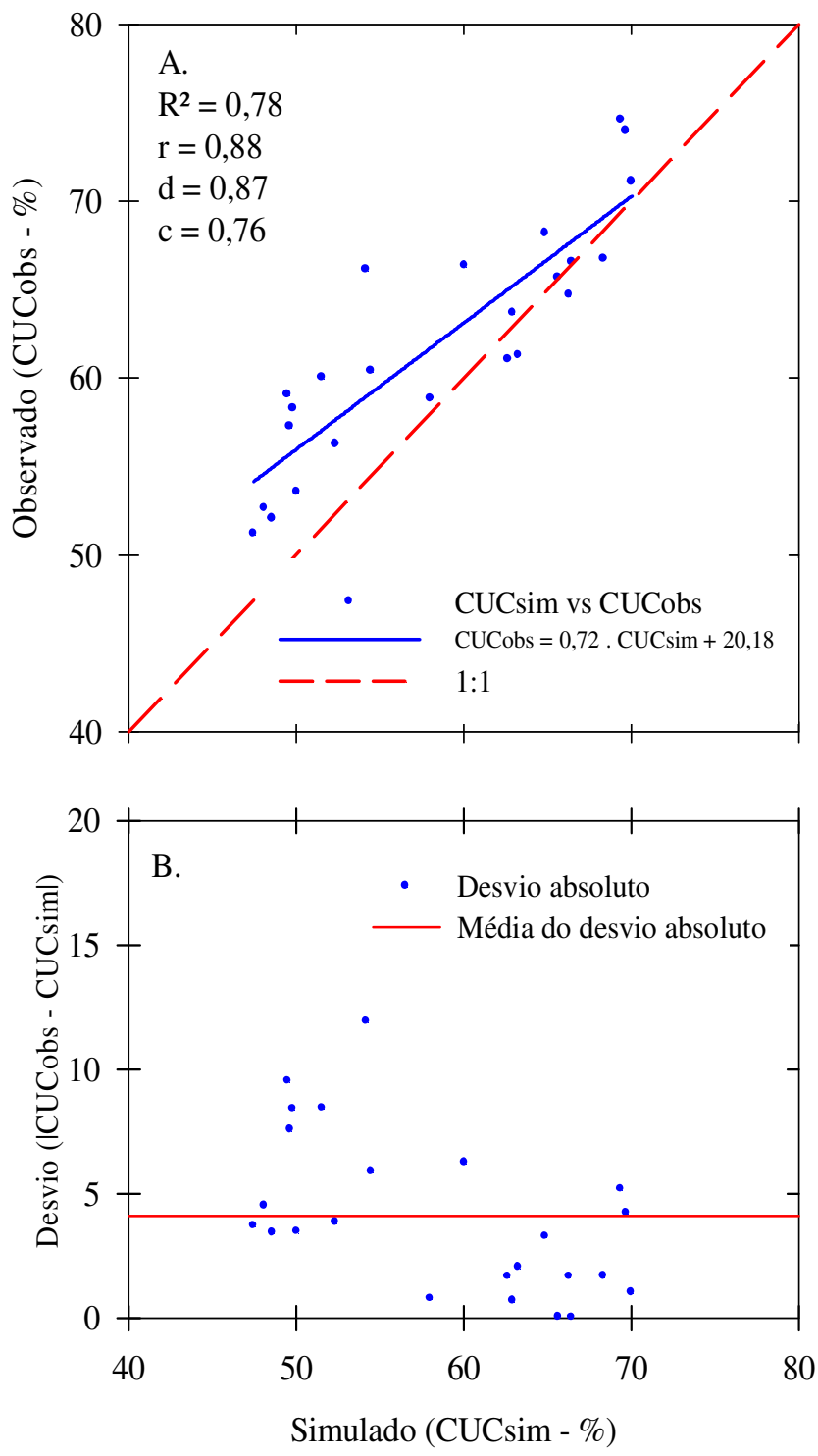

Figura 8. Valores de Coeficiente de Uniformidade de Christiansen: (A) simulados vs. observados e (B) diferenças absolutas (\%) entre estimativas de CUC observados $\left(C \cup C_{\text {obs }}\right)$ e simulados $\left(\mathrm{CUC}_{\text {sim }}\right)$, em função dos valores de $\mathrm{CUC}_{\text {sim }}$

Na Figura 8B se observam os 24 valores absolutos (Desvios) da diferença entre valores de CUC, oriundos de simulações e de ensaios de campo, para os aspersores Agropolo/NY e Naan/ 5024; nesta figura o valor médio das diferenças absolutas $(4,1 \%)$ é bastante similar ao obtido por Faria et al. (2009) para o ajuste do modelo de Richards \& Weatherhead (1993) aplicado para o canhão hidráulico Plona/R1250 e se encontra abaixo do limite de $5 \%$ considerado, por Montero et al. (2001), muito bom.

\section{CONCLusões}

1. Os valores ajustados dos seis parâmetros empíricos do modelo de Richards \& Weatherhead (1993) permitiram reproduzir, de forma satisfatória, a distorção do padrão de distribuição de água, sendo o modelo capaz de fornecer 
estimativas adequadas do Coeficiente de Uniformidade de Christiansen para aspersores de tamanho médio, operando isoladamente e em linhas laterais, sob diferentes condições de vento.

2. O bom desempenho das simulações permite inferir que o trabalho de campo requerido para produzir dados de uniformidade de aplicação de água de aspersores de tamanho médio, sob diferentes condições de vento, pode ser simplificado pela utilização do modelo de Richards \& Weatherhead (1993).

\section{LITERATURA CITADA}

Beskow, S.; Faria, L. C.; Colombo, A.; Moura, D. C. M. Modelagem das perdas de água por evaporação e arraste em aspersores de média pressão. Revista Brasileira de Engenharia Agrícola e Ambiental, v.15, p.221-228, 2011.

Burden, R. L.; Faires, J. D. Análise numérica. São Paulo: Thomson Learning, 2003. 736p.

Camargo, A. P. de; Sentelhas, P. C. Avaliação do desempenho de diferentes métodos de estimativa da evapotranspiração potencial no estado de São Paulo, Brasil. Revista Brasileira de Agrometeorologia, v.5, p.89-97, 1997.

Carrión, P.; Tarjuelo, J. M; Montero J. SIRIAS: A simulation model for sprinkler irrigation. Part I: Description of model.Irrigation Science, v.20, p.73-84, 2001.

Coelho, R. D.; Folegatti, M. V. Influência do nível da tensão da mola na percentagem de água desviada e de energia absorvida em um aspersor de impacto. Revista Brasileira de Engenharia Agrícola e Ambiental, v.2, p.170-174, 1998.

Conceição, M. A. F.; Coelho, R. D. Simulating wind effect on microsprinkler water distribution. Scientia Agricola, v.60, p.205-209, 2003.

Duan, Q.; Sorooshian, S.; Gupta, V. Effective and efficient global optimization for conceptual rainfall-runoff models. Water Resources Research, v.28, p.1015-1031, 1992.

Faria, L.C. Modelagem da aplicação de água de aspersores de tamanho médio operando em condições de vento. Lavras: UFLA, 2011.81p. Tese Doutorado

Faria L.C.; Colombo, A.; Oliveira, H. F. E. de; Prado, G. Simulação da uniformidade da irrigação de sistemas convencionais de aspersão operando sob diferentes condições de vento. Engenharia Agrícola, v.28, p.427-437, 2009.

Granier, J.; Molle, B.; Deumier, J. M. IRRIPARC-Part 1: Modeling spatial water distribution under a sprinkler in windy conditions. In: European Regional Conference of Theinternational Commission on Irrigation and Dreinage, 20, 2003, Montpellier. Anais... Montpellier: AFEID, 2003. CD-Rom
Holzapfel, E. A.; Prado, X. M.; Paz, V. P. S.; Rodriguez, A.; Orrego, X. C. Lopez, M. A. Análises técnico-econômico para selección de aspersores. Revista Brasileira de Engenharia Agrícola e Ambiental, v.11, p.557-563, 2007.

ISO - InternationalOrganization for Standardization. ISO 77492. Irrigation equipment - Rotating sprinklers - part 2: Uniformity of distribution and test methods, 1990. 6p.

Justi, A. L., Vilas Boas, M. A., Sampaio, S. C. Índice de capacidade do processo na avaliação da irrigação por aspersão. Engenharia Agrícola, v.30, p.264-270, 2010.

Montero, J.; Tarjuelo, J. M.; Carrión, P. SIRIAS: Asimulation model for sprinkler irrigation. Part II: Calibration and validation of the model. Irrigation Science, v.20, p.85-98, 2001.

Newell, G. Traveling gun simulation model TRAVGUN: User's manual and technical documentation. Toowoomba: National Centre for Engineering in Agriculture-NCEA. University of Southern Queensland-USQ, 2003. 89p.

Oliveira, F. E. de; Colombo, A.; Faria, L. C. Modelagem dos efeitos do vento sobre as dimensões do alcance do joto de um canhão hidráulico. Revista Brasileira de Engenharia Agrícola e Ambiental, v.13, p.818-824, 2009.

Ortega, J. F.; Tarjuelo, J. M.; Montero, J.; Juan, J. A. Discharge efficiency in sprinkling irrigation: analysis of the evaporation and drift losses in semi-arid areas. Agricultural Engineering International, the CIGR Ejournal, v.2, p.1-21, 2000.

Plaýan, E., Zapata, N.; Faci, J. M.; Tolosa, D.; Lacuerva, J. L. Pelegri, J.; Salvador, R.; Sanches, I.; Lafita, A. Assessing sprinkler irrigation uniformity using a ballistic simulation model. AgriculturalWater Management, v.84, p.89-100, 2006.

Prado, G.; Colombo A. Distribuição espacial de água aplicada por equipamentos autopropelidos de irrigação - Parte I: Modelagem com o simulasoft. Irriga, v.15, p.51-62, 2010a.

Prado, G.; Colombo A. Distribuição espacial de água aplicada por equipamentos autopropelidos de irrigação - Parte II: Validação do Simulasoft. Irriga, v.15, p.63-74, 2010 b.

Richards, P. J.; Weatherhead, E. K. Prediction of raingun application patterns in windy conditions. Journal of Agricultural Engineering Research, v.54, p.281-291. 1993.

Smith, R.J.; Gillies, M.H.; Newell, G.; Foley, J.P. A decision support model for travelling gun irrigation machines, Biosystems Engineering, v.100, p.126-136, 2008.

Solomon, K. Variability of sprinkler coefficient of uniformity test results. Transactions of the American Society of Agricultural Engineers, v.22, p.1078-80-1086, 1979.

Vories, E. D.; Ace, S. M.; Bernuth, R. D. von. Simulating sprinkler performance in wind. Journal of Irrigation and Drainage Engineering, v.113, p.119-130, 1987. 